\title{
DESENVOLVIMENTO E NUTRIÇÃO DO GIRASSOL COM LODO DE ESGOTO E NITROGÊNIO
}

\author{
Thomaz Figueiredo Lobo* \\ Helio Grassi Filho** \\ Leonardo Theodoro Bull*** \\ Ana Carolina Barbosa Kummer ${ }^{* * * *}$ \\ Flavia Luize Pereira de Souza*****
}

RESUMO: O objetivo deste trabalho foi avaliar a influência do lodo de esgoto compostado e do nitrogênio nos fatores nutricionais e no desenvolvimento do girassol após a sexta aplicação em rotações de culturas. O experimento foi conduzido na Fazenda Experimental São Manuel, pertencente à Faculdade de Ciências Agronômicas da UNESP de Botucatu (SP). Foi adotado o delineamento experimental em blocos casualizados, constituídos por 6 tratamentos e 4 repetições assim definidos: T0 sem adubação nitrogenada; T1 - adubação sintética nitrogenada; T2 - 50\% adubação nitrogenada proveniente do lodo de esgoto e 50\% na forma sintética; T3 - 100\% adubação nitrogenada proveniente do lodo de esgoto; T4 - 150\% adubação nitrogenada proveniente do lodo de esgoto; T5 - 200\% adubação nitrogenada proveniente do lodo de esgoto. O lodo de esgoto compostado proporcionou um incremento nos teores foliares de N, P, S, Fe, Mn e Zn e uma maior exportação de todos os nutrientes. A adubação química nitrogenada proporcionou decréscimo de K e um aumento do B, Fe e Mn na folha e aumento na exportação de todos os nutrientes. O lodo de esgoto compostado e o fertilizante nitrogenado proporcionaram um aumento na altura de plantas, diâmetro de haste, número de folhas e diâmetro de capítulo.

PALAVRAS-CHAVE: Agricultura orgânica; Exportação de nutrientes; Fatores bióticos; Sustentabilidade.

\footnotetext{
"Pós-doutorado pela Faculdade de Agronomia - UNESP no departamento de irrigação e drenagem. Docente da Universidade Sagrado Coração, Brasil. E-mail: thomaz.lobo@superig.com.br

** Docente Titular da Universidade Estadual Paulista Júlio de Mesquita Filho, Faculdade de Ciências Agronômicas, Departamento de Recursos Naturais - Ciência do Solo, Brasil.

${ }^{*}$ Docente Titular em Fertilidade do Solo pela UNESP Botucatu. Departamento recursos naturais e ciência do solo. Brasil.

${ }^{* * * * *}$ Doutorado em Agronomia - Irrigação e Drenagem - pela FCA/UNESP, docente na Universidade Estadual de Ponta Grossa, Departamento de Engenharia Civil. Brasil.

*******a Universidade Sagrado Coração. Brasil.
} 


\section{DEVELOPMENT OF NUTRITION OF SUNFLOWER WITH SEWAGE SLUDGE AND NITROGEN}

ABSTRACT: The influence of composted sewage sludge and nitrogen in nutrition factors and the development of sunflower crops after the sixth application in crop rotation are evaluated. The experiment was conducted on the Experimental Farm São Manuel, Faculty of Agronomic Sciences of UNESP in Botucatu SP Brazil. Experimental design comprised randomized blocks with 6 treatments and 4 replications: T0 - without nitrogen fertilization; T1 - synthetic nitrogen fertilization; T2 - 50\% nitrogen fertilization derived from sewage sludge and 50\% in synthetic form; T3 - 100\% nitrogen fertilization from sewage sludge; T4 - 150\% nitrogen fertilization from sewage sludge; $\mathrm{T} 5$ - 200\% nitrogen fertilization from sewage sludge. Composted sewage sludge increased $\mathrm{N}, \mathrm{P}, \mathrm{S}, \mathrm{Fe}, \mathrm{Mn}$ and $\mathrm{Zn}$ rates in leaves and a greater export of all nutrients. Chemical nitrogen fertilization decreased $\mathrm{K}$ and increased $\mathrm{B}, \mathrm{Fe}$ and $\mathrm{Mn}$ in the leaf and an increase in the export of all nutrients. Composted sewage sludge and nitrogen fertilizer increased plants' height, stalk diameter, number of leaves and chapter diameter.

KEY WORDS: Organic agriculture; Export of nutrients; Biotic factors; Sustainability.

\section{INTRODUÇÃO}

No Brasil, a disposição final do lodo geralmente é o aterro sanitário. Além do alto custo, que pode chegar a 50\% do custo operacional de uma Estação de Tratamento de Esgoto - ETE, a disposição de um resíduo com elevada carga orgânica no aterro agrava ainda mais o problema com o manejo do lixo urbano. Em países da Europa e América do Norte, o lodo geralmente é incinerado, depositado em aterros sanitários ou utilizado em áreas agrícolas, dependendo das características do resíduo. Na maioria dos países existem normas que regulamentam o destino do lodo, garantindo uma disposição segura. A adição ao solo parece ser a melhor opção sob o ponto de vista econômico e ambiental, uma vez que apresenta o menor custo e promove a reciclagem de matéria orgânica e nutrientes (BETTIOL; CAMARGO, 2007).

A utilização de lodo de esgoto é uma prática altamente promissora para o 
desenvolvimento de sistemas agrícolas sustentáveis. No entanto, normas rígidas pela elaboração de projetos devem ser seguidas para minimizar o impacto desta prática no ambiente, a norma técnica P4.240 (CASTRO; OLIVEIRA, 2005).

A utilização do lodo de esgoto em solos agrícolas tem como principal benefício a incorporação dos macronutrientes ( $\mathrm{N}$ e $\mathrm{P}$ ) e dos micronutrientes ( $\mathrm{Zn}$, $\mathrm{Cu}, \mathrm{Fe}, \mathrm{Mn}$ e Mo) (BETIOL; CAMARGO, 2007).

Para o girassol as recomendações de adubação nitrogenada de cobertura variam de 40 a $80 \mathrm{~kg} \mathrm{ha}^{-1}$ de $\mathrm{N}$. Como ainda não se estabeleceu correlação entre teores disponíveis do solo e a produtividade, a definição das doses de $\mathrm{N}$ a aplicar baseia-se na produtividade esperada (CANTARELLA, 1985).

O N é o segundo nutriente mais requerido pela cultura do girassol, acumulando $130 \mathrm{~kg} \mathrm{ha}^{-1}$, atrás somente do $\mathrm{K}$, porém grande quantidade de $\mathrm{K}$ é retornada ao solo, através dos restos culturais. Nos tecidos, a concentração de $\mathrm{N}$ varia dependendo do genótipo, de 35 a $50 \mathrm{~g} \mathrm{~kg}^{-1}$ nas folhas e de 4 a $10 \mathrm{~g} \mathrm{~kg}^{-1}$ no caule, no período de início de florescimento e o enchimento dos grãos.

O objetivo deste trabalho foi avaliar a influência de doses de lodo de esgoto compostado e do manejo de $\mathrm{N}$ nos fatores nutricionais e desenvolvimento do girassol.

\section{MATERIAL E MÉTODOS}

O experimento foi conduzido na Fazenda Experimental São Manuel, pertencente à Faculdade de Ciências Agronômicas da UNESP de Botucatu (SP), localizada no município de São Manuel a $22^{\circ} 25^{\prime}$ Latitude Sul, $48^{\circ} 34^{\prime}$ Longitude Oeste, com altitude de 750 metros.

Antecedendo a instalação do experimento, foram cultivados dois ciclos consecutivos de girassol semeados em dezembro de 2004 e novembro de 2005 , respectivamente, com aplicações de lodo de esgoto e cultivado com trigo e triticale, avaliando o efeito residual dessas duas aplicações de lodo de esgoto, em abril de 2006. Foi cultivada em março de 2007 a cultura da aveia com doses de lodo de esgoto com intuito de fornecer palha para iniciar no sistema de plantio direto. Em dezembro 
de 2007 foi aplicado o lodo de esgoto compostado em sistema de semeadura direta para o cultivo do feijão comum. Em maio de 2008 com aplicação de lodo compostado foi cultivado triticale.

Em dezembro de 2008 foi semeado o girassol deste experimento. A cultivar utilizada foi a HELIO 251, fornecida pela empresa Helianthus do Brasil, que é um híbrido simples.

Foi adotado o delineamento experimental em blocos casualizados, constituídos por 6 tratamentos e 4 repetições assim definidos: T0 - sem adubação nitrogenada; T1 - adubação química nitrogenada de acordo com Raij et al. (1997); T2 - 50\% adubação nitrogenada proveniente do lodo de esgoto compostado e 50\% na forma mineral; T3 - 100\% adubação nitrogenada proveniente do lodo de esgoto compostado; T4 - 150\% adubação nitrogenada proveniente do lodo de esgoto compostado; T5 - 200\% adubação nitrogenada proveniente do lodo de esgoto compostado.

Cada parcela constou de uma área de 100 metros quadrados $(14$ x 7,2 m) com um espaço de $3 \mathrm{~m}$ entre parcelas do mesmo bloco. O espaço de um bloco a outro foi de $1,8 \mathrm{~m}$.

$\mathrm{O}$ composto de lodo de esgoto apresenta as seguintes características: $\mathrm{N}$ 2,44 $\mathrm{g} \mathrm{kg}^{-1}, \mathrm{P}_{2} \mathrm{O}_{5}-2,0 \mathrm{~g} \mathrm{~kg}^{-1} ; \mathrm{K}_{2} \mathrm{O} 0,4 \mathrm{~g} \mathrm{~kg}^{-1}$; umidade 58\%; $\mathrm{C}$ 29,4 $\mathrm{g} \mathrm{kg}^{-1}, \mathrm{Ca}-1,9 \mathrm{~g}$ $\mathrm{kg}^{-1} ; \mathrm{Mg}-0,3 \mathrm{~g} \mathrm{~kg}^{-1} ; \mathrm{S}-1,6 \mathrm{~g} \mathrm{~kg}^{-1} ; \mathrm{Na}-1340 \mathrm{mg} \mathrm{kg}{ }^{-1} ; \mathrm{Cu}-304 \mathrm{mg} \mathrm{kg}^{-1} ; \mathrm{Fe}-23.250 \mathrm{mg}$ $\mathrm{kg}^{-1} ; \mathrm{Mn}-472 \mathrm{mg} \mathrm{kg}^{-1} ; \mathrm{Zn}-3750 \mathrm{mg} \mathrm{kg}^{-1} ; \mathrm{C} / \mathrm{N}$ - 12/1; $\mathrm{pH}$ - 6,6; a metodologia utilizada foi de Lanarv (1988).

Antes da semeadura do girassol foi aplicado o Gliphosate (ia), na dosagem de $2 \mathrm{~L} \mathrm{ha}^{-1}$, juntamente com o ácido bórico $(17 \% \mathrm{~B})$, na dosagem de $6 \mathrm{~kg} \mathrm{ha}^{-1}$, em um volume de calda de $320 \mathrm{~L} \mathrm{ha}^{-1}$, objetivando o controle de plantas daninhas e fornecimento de B para a cultura, elemento imprescindível para o girassol.

As dosagens de lodo de esgoto compostado a base seca foram: $3.682,6 \mathrm{~kg}$ $\mathrm{ha}^{-1} ; 7.365,1 \mathrm{~kg} \mathrm{ha}^{-1} ; 11.047,6 \mathrm{~kg} \mathrm{ha}^{-1} ; 14.730,2 \mathrm{~kg} \mathrm{ha}^{-1}$, respectivamente nos tratamentos T2, T3, T4 e T5. Foi calculado em função das doses de N com mineralização de 30\% (CASTRO; OLIVEIRA, 2005), correspondendo as dosagens de $\mathrm{N}$ de 27, 54, 81 e $108 \mathrm{~kg} \mathrm{ha}^{-1}$ nos tratamentos T2, T3, T4 e T5 respectivamente.

Foi semeado em um espaçamento de 0,7 metros entre linha e 3,5 sementes 
por metro, totalizando um estande de 50.000 plantas em um ha, na profundidade de $3 \mathrm{~cm}$. A adubação de plantio para todas as parcelas foi constituída de $51 \mathrm{~kg} \mathrm{ha}^{-1}$ de $\mathrm{P}_{2} \mathrm{O}_{5}$, na forma de superfosfato simples $\left(170 \mathrm{~g} \mathrm{~kg}^{-1} \mathrm{P}_{2} \mathrm{O}_{5}\right)$, e $30,5 \mathrm{~kg} \mathrm{ha}^{-1}$ de $\mathrm{K}_{2} \mathrm{O}$, na forma de cloreto de potássio ( $600 \mathrm{~g} \mathrm{~kg}^{-1} \mathrm{~K}_{2} \mathrm{O}$ ). A adubação de $\mathrm{N}$ foi realizada 12 dias após a semeadura no T1, na dosagem de $10 \mathrm{~kg} \mathrm{ha}^{-1}$ de N, na forma de ureia (450 g $\mathrm{kg}^{-1}$ de N), na fase em que o girassol começou a emergir.

No dia da semeadura foi efetuada também uma aplicação com Glyphosate na dosagem de $1 \mathrm{~kg} \mathrm{ha}^{-1}$, com um volume de calda de $300 \mathrm{~L} \mathrm{ha}^{-1}$.

Os tratamentos que receberam a adubação de cobertura nitrogenada foram os tratamentos T1 e T2. O tratamento T1 recebeu $40 \mathrm{~kg} \mathrm{ha}^{-1}$ de $\mathrm{N}$, que é o recomendado para o Estado de São Paulo, de acordo com o Boletim 100 (RAIJ et al., 1997); o tratamento T2 recebeu $25 \mathrm{~kg} \mathrm{ha}^{-1}$ de N, pois os outros $25 \mathrm{~kg} \mathrm{ha}^{-1}$ foram aplicados antes da semeadura na forma de lodo de esgoto, totalizando os $50 \mathrm{~kg} \mathrm{ha}^{-1}$, que é o recomendado para o Estado de São Paulo. A fonte utilizada para a adubação foi a ureia que contém $450 \mathrm{~g} \mathrm{~kg}^{-1}$ de N. Essa operação foi executada aos 30 dias após a emergência.

Para ser avaliada a diagnose nutricional foram coletadas as terceiras e quartas folhas contando de cima para baixo em 10 plantas de cada parcela na época do florescimento. Estas folhas foram lavadas com água comum, detergente e água deionizada. Em seguida foram postas para secar a $60^{\circ} \mathrm{C}$ na estufa até atingir o peso constante, após retirar da estufa estas amostras foram moídas. Nos aquênios do girassol foi feito o mesmo procedimento. O material moído foi acondicionado em sacos de papel e encaminhado para análise no Laboratório de Nutrição Mineral de Plantas do Departamento de Recursos Naturais/Ciência do Solo da FCA/UNESP. As amostras foram analisadas para os teores de N, P, K, Ca, Mg, S, B, Cu, Fe, Mn e Zn, seguindo a metodologia de rotina do referido laboratório (MALAVOLTA et al., 1997).

Com os teores das sementes foi multiplicado pela produtividade do girassol com isto tem-se a exportação de nutrientes.

No decorrer do experimento, foram coletados altura por planta, número de folhas e diâmetro de haste, até a fase de florescimento. A altura de plantas, número de folhas e diâmetro de haste em 10 plantas, distribuída aleatoriamente por parcela. As medidas de altura de plantas foram realizadas aos 40, 46, 61 e 68 dias após a 
semeadura. Durante o desenvolvimento vegetativo, a altura foi determinada pela medida do solo até a inserção da última folha totalmente lançada; na fase reprodutiva, do nível do solo até a inserção do capítulo. Para o diâmetro de haste foi efetuado com paquímetro a $2 \mathrm{~cm}$ do solo, estas medidas foram efetuadas aos 46, 61 e 68 dias da semeadura. O número de folhas foi contado aos 61 dias após a semeadura. Esse parâmetro foi determinado pela contagem das folhas totalmente abertas.

$\mathrm{Na}$ colheita avaliaram-se altura de plantas, diâmetro da haste e diâmetro de capítulo.

Os dados climatológicos do local de ensaio estão na Figura 1 e referem-se à precipitação pluviométrica $(\mathrm{mm})$ e temperatura.
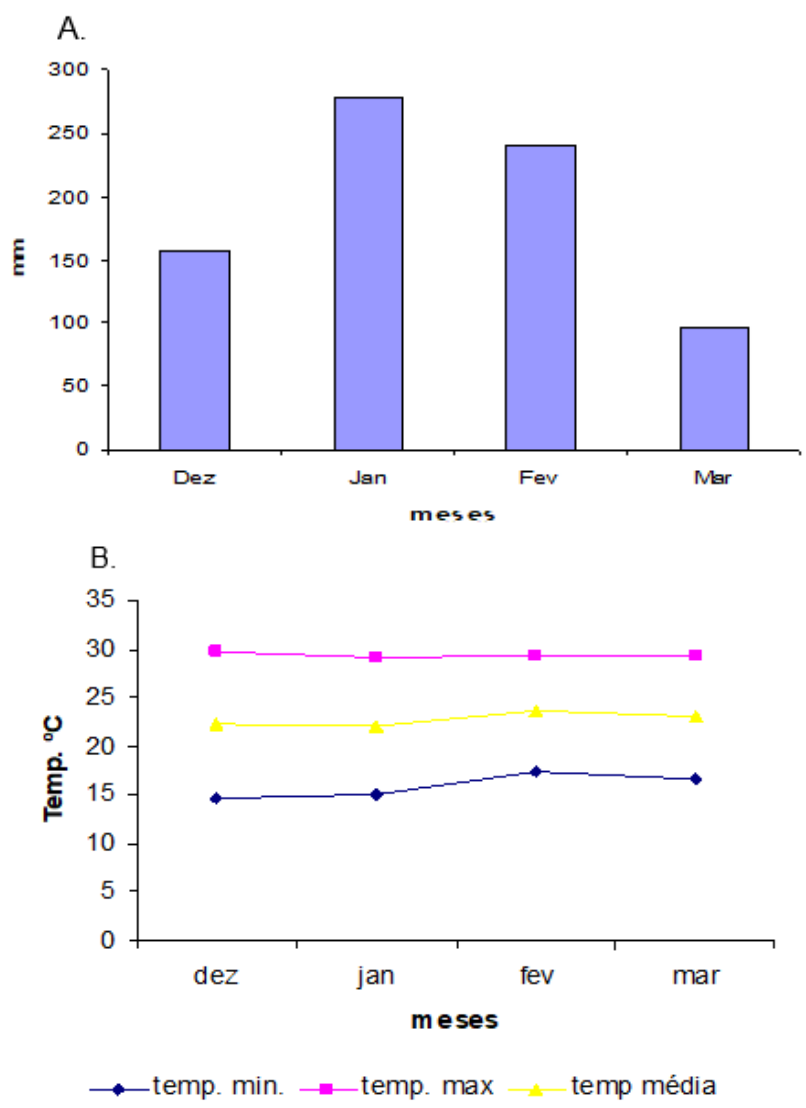

Figura 1. Precipitações médias (A), temperatura mínima média, máxima média e média no período do ciclo do girassol no ano 2008/2009 (B).

Fonte: Elaborada pelos autores. 
Os resultados foram submetidos a análise de variância e as médias comparadas pelo teste de Duncan a $5 \%$ de probabilidade. Os resultados coletados nos tratamentos T0, T3, T4 e T5 foram tratados pela análise de regressão da variância.

\section{RESULTADO E DISCUSSÃO}

Segundo Castro e Oliveira (2005), os teores foliares de N, P, K, Ca, Mg, S, considerados adequados para a cultura do girassol são respectivamente $35-50 \mathrm{~g} \mathrm{~kg}$ 1, 2,9 - 4,5 $\mathrm{g} \mathrm{kg}^{-1}, 31$ - $45 \mathrm{~g} \mathrm{~kg}^{-1}, 19$ - $32 \mathrm{~g} \mathrm{~kg}^{-1}, 5,1$ - 9,4 $\mathrm{g} \mathrm{kg}^{-1}, 3$ - 6,4 $\mathrm{g} \mathrm{kg}^{-1}$.

Tabela 1. Teores foliares de macronutrientes e micronutrientes no girassol

\begin{tabular}{|c|c|c|c|c|c|c|}
\hline \multicolumn{7}{|c|}{ Macronutrientes } \\
\hline Tratamentos & $\mathrm{N}$ & $\mathrm{P}$ & $\mathrm{K}$ & $\mathrm{Ca}$ & $\mathrm{Mg}$ & $\mathrm{S}$ \\
\hline \multicolumn{7}{|c|}{ (2) } \\
\hline T0 & $40,9 \mathrm{c}$ & $4,8 \mathrm{c}$ & $44,7 \mathrm{a}$ & $35,8 \mathrm{a}$ & 6,8 & $3,21 \mathrm{~b}$ \\
\hline $\mathrm{T} 1$ & $43,0 \mathrm{bc}$ & $4,8 \mathrm{c}$ & $39,5 \mathrm{c}$ & $33,8 \mathrm{ab}$ & 7,2 & $3,44 b$ \\
\hline $\mathrm{T} 2$ & $43,9 \mathrm{ab}$ & $5,4 \mathrm{~b}$ & $44,0 \mathrm{ab}$ & $29,0 \mathrm{~d}$ & 7,3 & $3,77 \mathrm{~b}$ \\
\hline T3 & $43,3 \mathrm{bc}$ & $5,5 \mathrm{ab}$ & 40,2 bc & $31,1 \mathrm{bcd}$ & 7,3 & $4,50 \mathrm{a}$ \\
\hline T4 & $46,7 \mathrm{a}$ & $5,8 \mathrm{ab}$ & $42,25 \mathrm{abc}$ & $30,1 \mathrm{~cd}$ & 6,8 & $4,55 \mathrm{a}$ \\
\hline $\mathrm{T} 5$ & $45,3 \mathrm{ab}$ & $5,9 \mathrm{a}$ & $38,92 \mathrm{c}$ & $32,8 \mathrm{abc}$ & 7,6 & $5,11 \mathrm{a}$ \\
\hline $\mathrm{F}$ & 4,80 & 13,05 & 4,00 & 6,52 & 0,40 & 12,33 \\
\hline Média & 43,9 & 5,4 & 41,65 & 32,11 & 7,17 & 4,10 \\
\hline $\mathrm{CV}$ & 4,20 & 4,87 & 6,63 & 6,18 & 13,75 & 10,24 \\
\hline \multicolumn{7}{|c|}{ Micronutrientes } \\
\hline Tratamentos & B & $\mathrm{Cu}$ & $\mathrm{Fe}$ & $\mathrm{Mn}$ & $\mathrm{Zn}$ & \\
\hline \multicolumn{7}{|c|}{ 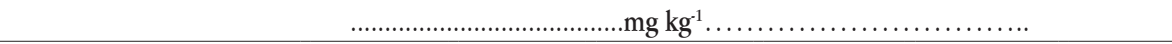 } \\
\hline T0 & $56,0 \mathrm{~b}$ & 40,2 & $78,0 \mathrm{~b}$ & $162,2 \mathrm{~b}$ & $62,2 \mathrm{c}$ & \\
\hline $\mathrm{T} 1$ & $85,3 \mathrm{a}$ & 33,7 & $114,2 \mathrm{a}$ & $250,7 \mathrm{a}$ & $63,0 \mathrm{c}$ & \\
\hline $\mathrm{T} 2$ & $69,3 \mathrm{ab}$ & 37,7 & $113,2 \mathrm{a}$ & $236,2 \mathrm{a}$ & $92,2 \mathrm{bc}$ & \\
\hline T3 & $57,1 \mathrm{~b}$ & 39,2 & $118,0 \mathrm{a}$ & $211,2 \mathrm{ab}$ & $115,0 \mathrm{~b}$ & \\
\hline T4 & $82,1 \mathrm{a}$ & 43,5 & $112,2 \mathrm{a}$ & $240,5 \mathrm{a}$ & $210,2 \mathrm{a}$ & \\
\hline $\mathrm{T} 5$ & $75,7 \mathrm{a}$ & 43,0 & $116,7 \mathrm{a}$ & $262,7 \mathrm{a}$ & $197,7 \mathrm{a}$ & \\
\hline $\mathrm{F}$ & 5,66 & 1,47 & 8,92 & 2,69 & 19,0 & \\
\hline Média & 70,9 & 39,6 & 108,7 & 227,3 & 123,9 & \\
\hline $\mathrm{CV}$ & 14,71 & 15,02 & 9,37 & 19,4 & 24,77 & \\
\hline
\end{tabular}

Médias seguidas pelas mesmas letras não diferem entre si pelo teste de Duncan 5\% de probabilidade. T0 - sem N, T1 -100\% AM, T2 - 50\% AM + 50\% LE, T3 - 100\% LE, T4 - 150\% LE, T5 - 200\% LE. AM - Adubação mineral; LE - Lodo de esgoto.

Fonte: Elaborada pelos autores 
A.

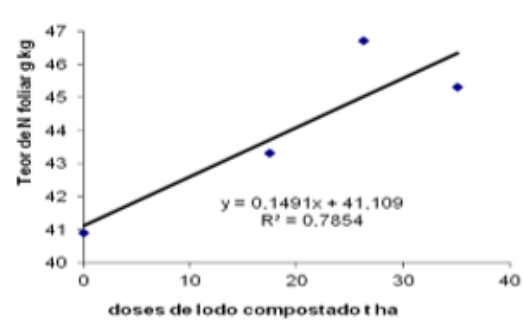

C.

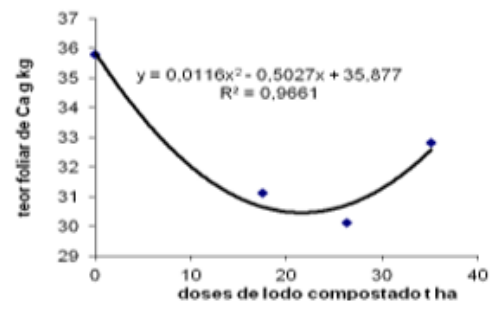

E.
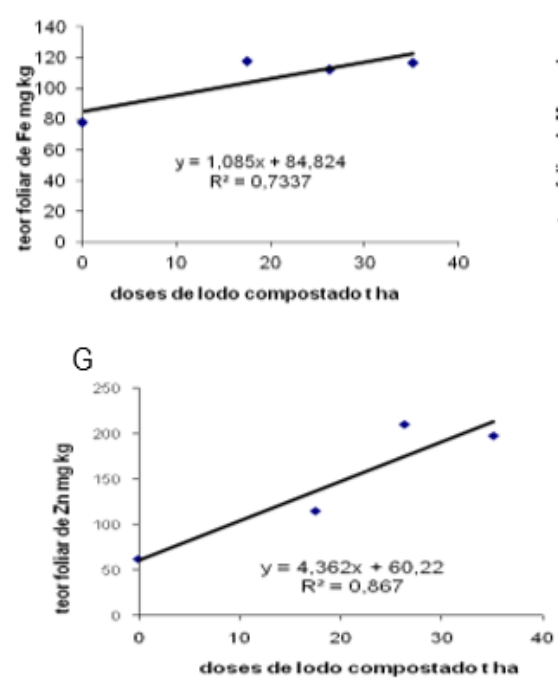

F.
B.

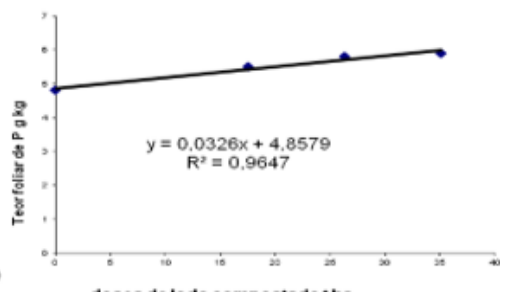

doses de lodo compostado tha
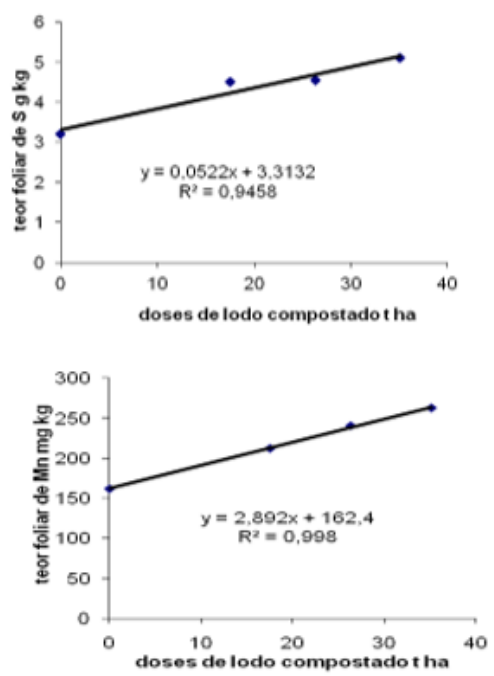

Figura 2. Teores foliares de macronutrientes (N (A), P (B), Ca (C), S (D)) e micronutrientes (Fe (E), Mn (F) e Zn (G) em função da dose de lodo compostado. Fonte: Elaborada pelos autores 
Pode-se verificar na Tabela 1 que para o teor de $\mathrm{N}$ todos os valores enquadram-se adequados. Entre os tratamentos o T4 foi superior em relação ao T0, T1 e T3. Verifica-se na Figura 2 que com o aumento da dose de lodo aumentou-se linearmente o teor de $\mathrm{N}$ na folha.

Quanto ao $\mathrm{P}$ pode ser verificado que todos os tratamentos apresentaram valores acima do adequado. Nos tratamentos que foi aplicado o lodo de esgoto compostado (T2, T3, T4 e T5) obtiveram teores de P na folha superiores aos T0 e T1. A Figura 2 ilustra tais informações que o aumento da dose de lodo proporcionou um incremento no teor de $\mathrm{P}$ nas folhas.

Todos os tratamentos apresentam teores foliares adequados de $\mathrm{K}$. O tratamento T0 obteve maior teor de $\mathrm{K}$ nas folhas que o T1, T2, T3 e T5 (Tabela 1). O aumento da dose de lodo não apresentou uma boa correlação com o teor foliar de K.

Os tratamentos que receberam lodo de esgoto (T2, T3, T4 e T5) apresentaram teores adequados de Ca, já os tratamentos que não receberam o lodo (T0 e T1) apresentaram acima do adequado. O T0 foi superior ao T2, T3 e T4, provavelmente pelo efeito diluição. Na Figura 2 verifica-se que houve um decréscimo do teor foliar de Ca com o aumento da dose de lodo compostado até certo momento e posteriormente ocorreu um acréscimo com o aumento da dose de lodo compostado. O decréscimo do teor de Ca nas folhas pode ter ocorrido em função do efeito da diluição, ou seja, a produção de massa da planta sem lodo era muito inferior do que as plantas adubadas com lodo por isso que ocorreu o decréscimo, se for verificado a quantidade total de Ca na planta teremos uma reta linear como aconteceu na exportação de Ca pelo girassol. O importante é que em nenhum tratamento obteve teores foliares de $\mathrm{Ca}$ deficiente.

Para o Mg todos os tratamentos apresentaram teores adequados e entre os tratamentos não ocorreram diferenças significativas e o aumento da dosagem de lodo não interferiu no teor de $\mathrm{Mg}$ na folha.

Todos os tratamentos apresentaram teores foliares de $S$ adequados, e entre os tratamentos o T3, T4 e T5 apresentaram superior aos outros tratamentos. Com o aumento da dose de lodo aumentou o teor de $S$ na folha de uma forma linear (Figura 2).

Nos teores de macronutrientes nas folhas de girassol praticamente não ocorreram diferenças, porém se for analisado o acúmulo destes nutrientes pode ter tido a diferença.

Lobo e Grassi Filho (2009), trabalhando com o lodo de esgoto em girassol, 
encontraram teores de macronutrientes inferiores aos obtidos no presente trabalho.

Segundo Castro e Oliveira (2005), os teores foliares de B, Cu, Fe, Mn, Zn, considerados adequados para a cultura do girassol são respectivamente 35 - $80 \mathrm{mg}$ $\mathrm{kg}^{-1}, 24$ - $42 \mathrm{mg} \mathrm{kg}^{-1}, 120$ - $235 \mathrm{mg} \mathrm{kg}^{-1}, 55$ - $180 \mathrm{mg} \mathrm{kg}^{-1}, 29$ - $43 \mathrm{mg} \mathrm{kg}^{-1}$.

Observando-se a Tabela 1 que os tratamentos T0, T2 e T3 apresentaram teores de B adequados e que T1, T4 e T5 apresentaram teores elevados. Os teores de B nos tratamentos T1, T4 e T5 foram superiores estatisticamente aos tratamentos T0 e T3 e que o T2 foi igual a todos os tratamentos. Com aumento da dose de lodo não houve uma interação com o teor foliar deste elemento.

$\mathrm{O}$ teor de $\mathrm{Cu}$ (Tabela 1) não diferiu estatisticamente e a média dos tratamentos apresentaram teores adequados e não teve influência com o aumento da dose de lodo compostado.

Todos os tratamentos apresentaram teores de Fe abaixo do adequado, este fato pode ser atribuído aos altos teores de $\mathrm{P}$ apresentados na folha e as quantidades elevadas de Mn. A elevada quantidade de P e Mn dificultam a mobilidade do Fe na planta (DECHEN; NACHTIGALL, 2006). O tratamento que não recebeu N (T0) obteve um teor de Fe inferior a todos os outros tratamentos. $\mathrm{O}$ aumento da dose de lodo compostado aumentou linearmente o teor de Fe nas folhas do girassol (Figura 2).

O T0 apresenta teores adequados de Mn e o restante todo acima do adequado. $\mathrm{O}$ T1 obteve um teor de Mn superior ao T0, tendo em vista que o $\mathrm{N}$ aumentou o teor de $\mathrm{Mn}$ nas folhas. O aumento da dose de lodo proporcionou um incremento no teor de Mn nas folhas como mostra a Figura 2.

Todos os tratamentos apresentaram teores elevados de $\mathrm{Zn}$ uma vez que quando iniciamos este ensaio os teores de Zn no solo já apresentaram índices altos. O T3 obteve um teor de $\mathrm{Zn}$ superior que T0 e T1 e os tratamentos que foram aplicados as maiores dose de lodo compostado (T4 e T5) obtiveram maiores teores de $\mathrm{Zn}$ que os outros tratamentos. $\mathrm{O}$ aumento da dose de lodo houve um incremento linear no teor de $\mathrm{Zn}$ nas folhas.

Comparando os dados obtidos em Lobo e Grassi Filho (2009), trabalhando com lodo de esgoto, obtiveram teores de Fe nas folhas mais elevados em relação a este trabalho e os outros micronutrientes apresentaram teores mais baixos e só houve variação para o $\mathrm{Zn}$, isto pode ser explicado por ter sido feita somente uma aplicação de lodo de esgoto. 
Tabela 2. Exportação de macronutrientes e micronutrientes do girassol

\begin{tabular}{|c|c|c|c|c|c|c|}
\hline \multicolumn{7}{|c|}{ Macronutrientes } \\
\hline \multirow[t]{2}{*}{ Tratamentos } & $\mathrm{N}$ & $\mathrm{P}$ & K & $\mathrm{Ca}$ & $\mathrm{Mg}$ & $S$ \\
\hline & \multicolumn{6}{|c|}{$\mathrm{kg} \mathrm{ha}^{-1}$} \\
\hline T0 & $44,01 \mathrm{~d}$ & $15,22 \mathrm{~d}$ & $20,04 \mathrm{c}$ & $2,67 \mathrm{~b}$ & $9,21 \mathrm{~d}$ & $3,08 \mathrm{~d}$ \\
\hline $\mathrm{T} 1$ & $102,56 \mathrm{bc}$ & $26,70 \mathrm{bc}$ & $38,21 \mathrm{~b}$ & $8,79 \mathrm{a}$ & $16,88 \mathrm{bc}$ & $5,86 \mathrm{bc}$ \\
\hline $\mathrm{T} 2$ & $123,67 \mathrm{ab}$ & $32,52 \mathrm{ab}$ & $41,00 \mathrm{~b}$ & $8,53 \mathrm{a}$ & $20,07 \mathrm{ab}$ & $6,86 \mathrm{ab}$ \\
\hline T3 & $84,93 \mathrm{c}$ & $23,67 c$ & $31,69 \mathrm{~b}$ & $6,25 \mathrm{a}$ & $14,28 \mathrm{c}$ & $4,77 \mathrm{c}$ \\
\hline $\mathrm{T} 4$ & $112,84 \mathrm{ab}$ & $28,41 \mathrm{bc}$ & $41,07 \mathrm{~b}$ & $7,02 \mathrm{a}$ & $17,83 \mathrm{bc}$ & $6,33 \mathrm{~b}$ \\
\hline $\mathrm{T} 5$ & $136,88 \mathrm{a}$ & $36,15 \mathrm{a}$ & $52,38 \mathrm{a}$ & $8,05 \mathrm{a}$ & $22,44 \mathrm{a}$ & $7,88 \mathrm{a}$ \\
\hline $\mathrm{F}$ & 16,26 & 14,62 & 9,37 & 7,91 & 12,81 & 14,42 \\
\hline Média & 100,82 & 27,11 & 37,40 & 6,88 & 16,79 & 5,79 \\
\hline $\mathrm{CV}$ & 16,24 & 13,93 & 18,90 & 23,51 & 15,43 & 15,29 \\
\hline \multicolumn{7}{|c|}{ Micronutrientes } \\
\hline \multirow[t]{2}{*}{ Tratamentos } & B & $\mathrm{Cu}$ & $\mathrm{Fe}$ & $\mathrm{Mn}$ & & \\
\hline & \multicolumn{6}{|c|}{$\mathrm{g} \mathrm{ha}^{-1}$} \\
\hline T0 & $51,00 \mathrm{c}$ & $33,76 \mathrm{~d}$ & $90,58 \mathrm{~d}$ & $52,71 \mathrm{c}$ & & \\
\hline $\mathrm{T} 1$ & $102,52 \mathrm{ab}$ & $77,17 \mathrm{bc}$ & $200,64 \mathrm{c}$ & $125,99 a$ & & \\
\hline $\mathrm{T} 2$ & $119,93 \mathrm{a}$ & $93,97 \mathrm{ab}$ & $309,71 \mathrm{ab}$ & $144,16 \mathrm{a}$ & & \\
\hline $\mathrm{T} 3$ & $78,85 \mathrm{bc}$ & $63,92 \mathrm{c}$ & $212,00 \mathrm{c}$ & $95,28 \mathrm{~b}$ & & \\
\hline $\mathrm{T} 4$ & $114,87 \mathrm{a}$ & 77,16 bc & $252,55 \mathrm{bc}$ & $125,29 \mathrm{a}$ & & \\
\hline $\mathrm{T} 5$ & $130,94 \mathrm{a}$ & $98,70 \mathrm{a}$ & $354,24 \mathrm{a}$ & $143,65 \mathrm{a}$ & & \\
\hline $\mathrm{F}$ & 2,40 & 4,89 & 2,41 & 4,03 & & \\
\hline Média & 55,03 & 74,11 & 238,07 & 114,51 & & \\
\hline $\mathrm{CV}$ & 21,93 & 15,74 & 20,63 & 15,84 & & \\
\hline
\end{tabular}

Médias seguidas pelas mesmas letras não diferem entre si pelo teste de Duncan 5\% de probabilidade. T0 - sem N, T1 -100\% AM, T2 - 50\% AM + 50\% LE, T3 - 100\% LE, T4 - 150\% LE, T5 - 200\% LE. AM - Adubação mineral; LE - Lodo de esgoto.

Fonte: Elaborada pelos autores

O tratamento $\mathrm{T} 5$ exportou mais $\mathrm{N}$ em relação aos tratamentos $\mathrm{T} 0$, T1 e T3. Os tratamentos T2 e T4 exportaram mais que o T0 e T3. O tratamento que não recebeu N (T0) foi o tratamento que menos exportou N (Tabela 2). O lodo proporcionou um aumento linear na exportação de N (Figura 3). Segundo Anjos (2009), para cada $1.000 \mathrm{~kg}$ de semente são exportados $23 \mathrm{~kg}$ de N, devido a esta constatação somente o tratamento que não recebeu $\mathrm{N}$ apresentou índice abaixo deste valor. 
A.

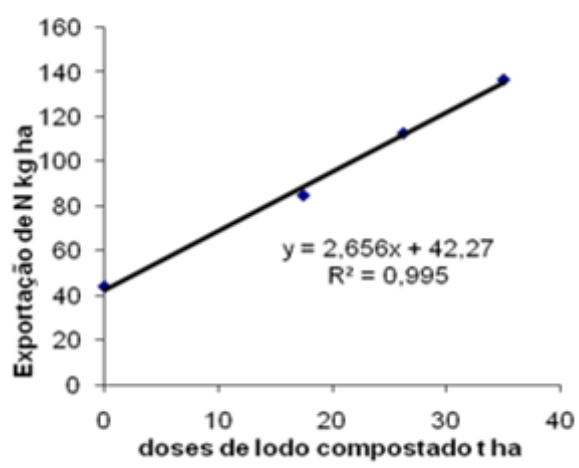

C.

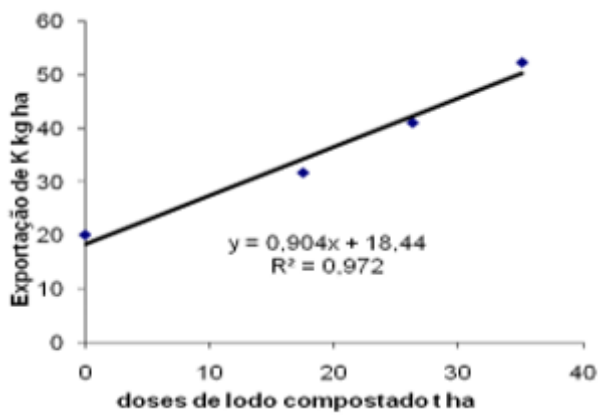

E.

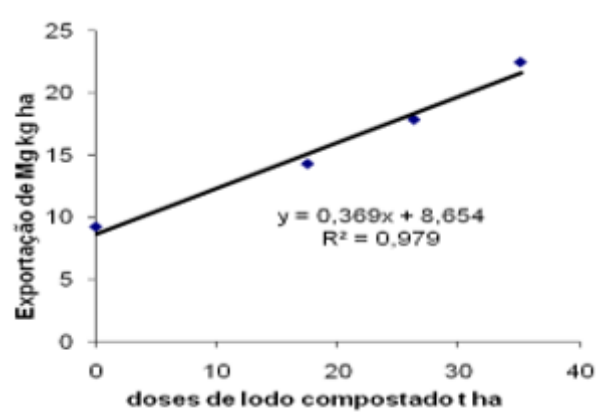

B.

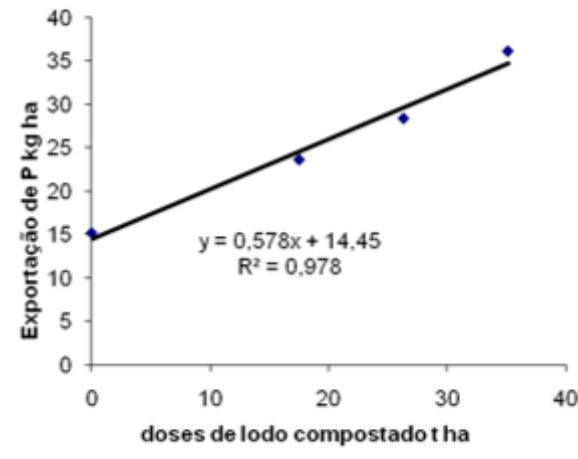

D.

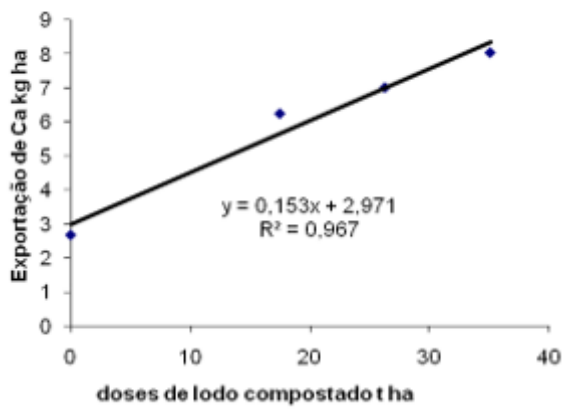

F.

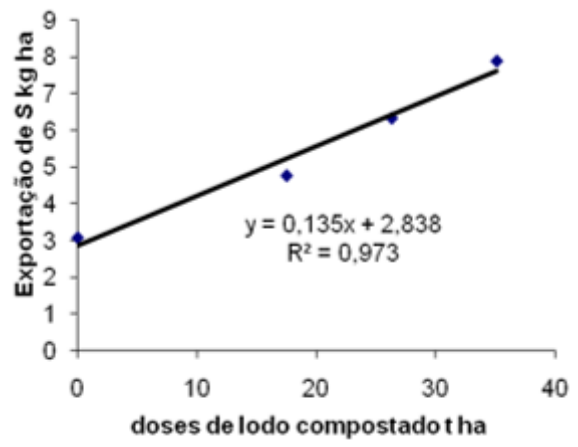


G.

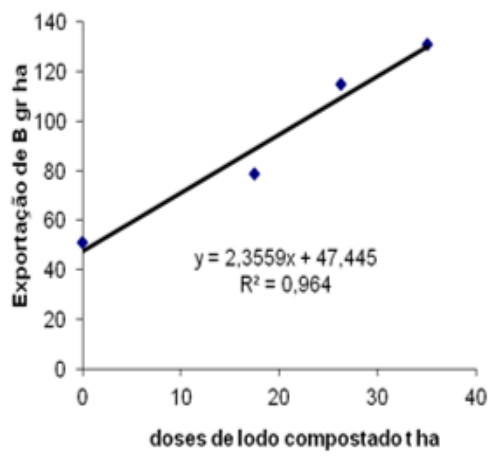

I.

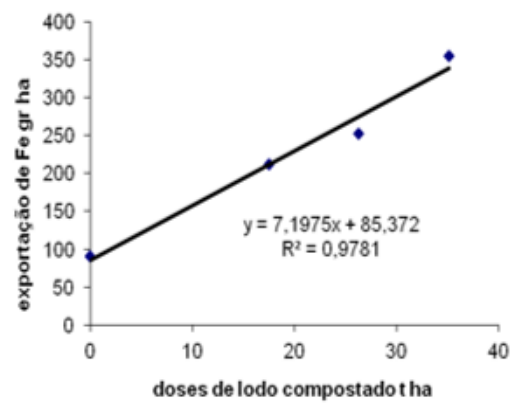

$\mathrm{K}$

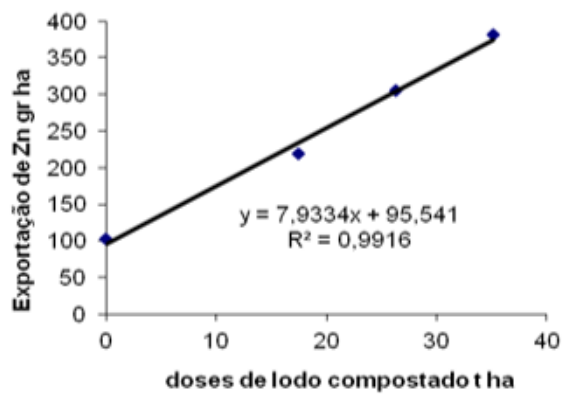

$\mathrm{H}$.

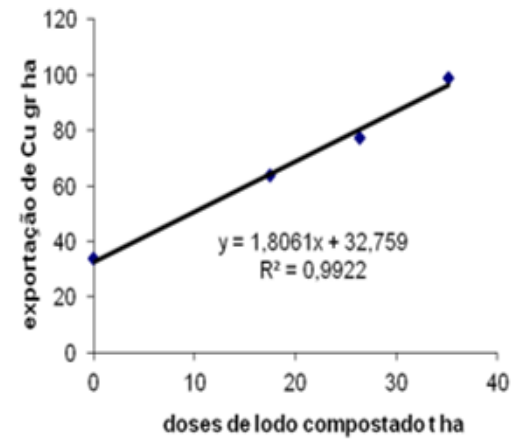

J.

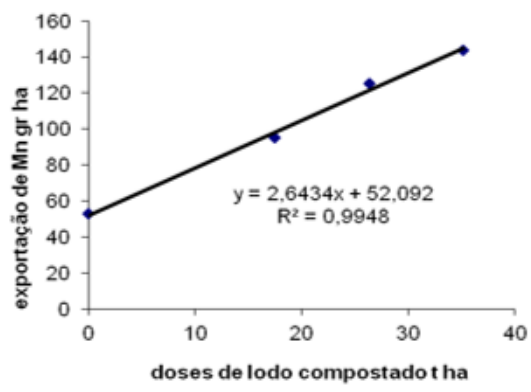

Figura 3. Exportação de macronutrientes no girassol em função da dose de lodo compostado: $N$ (A), P(B) K (C), Ca (D), Mg (E) e S (F) e de micronutrientes: B (G) Cu (H), Fe (I), Mn (J), Zn (K). Fonte: Elaborada pelos autores 
$\mathrm{Na}$ Tabela 2 verifica-se que a exportação de $\mathrm{P}$ pelo grão o T5 exportou maior quantidade de P que o T0, T1, T3 e T4. O tratamento que exportou menos P foi o tratamento que não recebeu $\mathrm{N}$ (T0). Na Figura 3 observa-se que à medida que aumentou a dose de lodo houve um incremento linear na exportação de P a cada 1.000 $\mathrm{kg}$ do composto aumentou $0,6 \mathrm{~kg}$ de $\mathrm{P}$ nos aquênios.

$\mathrm{O}$ T5 exportou mais K que todos os tratamentos e o T0, que não recebeu $\mathrm{N}$, exportou menos $\mathrm{K}$ em função da menor produtividade deste tratamento (Tabela 2). $\mathrm{Na}$ Figura 3 observa-se que com o aumento da dose de lodo aumentou linearmente a exportação de K: a cada $1.000 \mathrm{~kg}$ de lodo compostado aumentou $0,9 \mathrm{~kg}$ de $\mathrm{K}$ iniciando com 19,5 de K.

Para a exportação de Ca o tratamento que não recebeu N (T0) exportou menos Ca que os demais tratamentos (Tabela 2). Na Figura 3 observa-se que com o aumento da dose de lodo ocorreu um aumento linear na exportação de Ca: a cada $1.000 \mathrm{~kg}$ do composto exportou $153 \mathrm{~g}$ de Ca.

Verifica-se na Tabela 2 que o T5 exportou mais Mg que os tratamentos T0, T1, T3 e T4. O tratamento T0 foi o que menos exportou Mg. Verifica-se que o aumento da dose de lodo compostado ocorreu um aumento linear na exportação de $\mathrm{Mg}$ : a cada $1.000 \mathrm{~kg}$ do composto exportou $389 \mathrm{~g}$ de Mg (Figura 3).

A exportação de $S$ entre os tratamentos apresentados na Tabela 2 ocorreu na seguinte ordem: T5 $>$ T4 $>$ T3 $>$ T0 e o tratamento T1 não diferiu do T4 e do T3 e o T2 foi igual ao T4 e T5. Em função do aumento da dose de lodo ocorreu um aumento linear na exportação de $S$ : a cada $1.000 \mathrm{~kg}$ do composto aumentou $136 \mathrm{~g}$ de $S$ iniciando com 2,83 kg de $S$ (Figura 3).

Segundo Castro e Oliveira (2005) para cada $1000 \mathrm{~kg}$ de produção o girassol exporta $23 \mathrm{~kg}$ de N, $12 \mathrm{~kg}$ de $\mathrm{P}_{2} \mathrm{O}_{5}, 12 \mathrm{~kg}$ de $\mathrm{K}_{2} \mathrm{O}, 1,6 \mathrm{~kg}$ de Ca, 2,5 kg de $\mathrm{Mg}$ e 2,2 kg de $\mathrm{S}$, considerando esta informação o tratamento que não foi aplicado $\mathrm{N}$ apresentou em média um teor de $\mathrm{N}, \mathrm{K}, \mathrm{Ca}$ e $\mathrm{S}$ abaixo destes valores.

Verifica-se na Tabela 2 que o T2, T4 e T5 exportaram uma quantidade maior de B em relação ao T0 e ao T3. Observa-se na Figura 3 que com o aumento da dose de lodo compostado aumentou linearmente a exportação de B a cada $1.000 \mathrm{~kg}$ do composto de lodo de esgoto exportou 2,35 mg de B.

$\mathrm{Na}$ Tabela 2 verifica-se que para a exportação de $\mathrm{Cu}$ pelo grão o tratamento T5 foi superior ao T0, T1, T3 e o T4. O tratamento que não recebeu N (T0) foi inferior aos demais tratamentos. Houve um incremento linear da exportação deste elemento em função do aumento da dose de lodo compostado como mostra a Figura 3. 
O T5 exportou maior quantidade de Fe em relação aos tratamentos T0, T1, T3 e T4. O tratamento T0 foi o tratamento que menos exportou Fe (Tabela 2). Na exportação de Fe houve um incremento deste elemento em função do aumento da dose de lodo compostado (Figura 3), a cada $1.000 \mathrm{~kg}$ de lodo de esgoto aplicado exportou 7,17 mg de Fe iniciando com 88,37 mg de Fe.

Na Tabela 2 verifica-se que a exportação de Mn ocorreu na seguinte forma: T1, T2, T4 e T5 foram os tratamentos que mais exportaram e o tratamento que não recebeu N (T0) foi o que menos exportou. À medida que foi aumentando a dose de lodo compostado aumentou a exportação de Mn de uma forma linear como mostra a Figura 3.

Na Tabela 2 verifica-se que a exportação de Zn o T5 foi o tratamento que mais exportou. O T2 e o T4 foram os segundos tratamentos que mais exportaram. Os terceiros que mais exportaram foram o T1 e o T3 e o tratamento que não recebeu $\mathrm{N}$ (T0) foi o tratamento que menos exportou Zn. Com o aumento da dose de lodo compostado ocorreu um aumento linear na exportação de Zn, a cada $1.000 \mathrm{~kg}$ de composto orgânico adicionado 7,83 mg de Zn iniciando com 98,34 mg (Figura 3).

Segundo Castro e Oliveira (2005), para cada tonelada de grão o girassol exporta 23 gramas de $\mathrm{B}, 18$ gramas de $\mathrm{Cu}, 98$ gramas de $\mathrm{Fe}, 35$ gramas de $\mathrm{Mn}$ e 42 gramas de Zn. Somente no tratamento que não foi aplicado N (T0) apresentou um teor de $\mathrm{Cu}$ nas sementes inferior ao que Castro e Oliveira (2005) consideraram ideais.

Verifica-se que para o aumento da dose de lodo exportaram-se mais todos os nutrientes, ao observar um balanço geral pode ser visto que a quantidade colocada destes nutrientes pelo lodo apresentou-se muito superior em relação onde não foi colocado lodo.

Neste experimento as produtividades de grãos do girassol foram T0 - 2.074; T1 - 3.760; T2 - 4.524; T3 - 3.158; T4 - 4.009; T5 - $4.767 \mathrm{~kg} \mathrm{ha}^{-1}$, respectivamente (LOBO et al., 2013).

$\mathrm{Na}$ Tabela 3 apresentam-se as médias de alturas de plantas nos respectivos dias de semeadura 40, 46, 61 e 68. Observa-se que onde não se aplicou $\mathrm{N}$ apresentou-se sempre inferior estatisticamente em relação onde foi aplicado N. Dos tratamentos que foram aplicados $\mathrm{N}$ observa-se que onde se aplicou $\mathrm{N}$ mineral (100\%), apresentou-se em todos os momentos iguais estatisticamente onde se aplicou 200\% de $\mathrm{N}$ proveniente de composto de lodo de esgoto. Dos tratamentos que foram aplicados a mesma dose de N, o T1 apresentou-se superior aos demais. 
Tabela 3. Altura e diâmetro de haste médio de plantas de girassol em dias após a emergência e o número médio de folhas e de diâmetro médio do capítulo de plantas de girassol

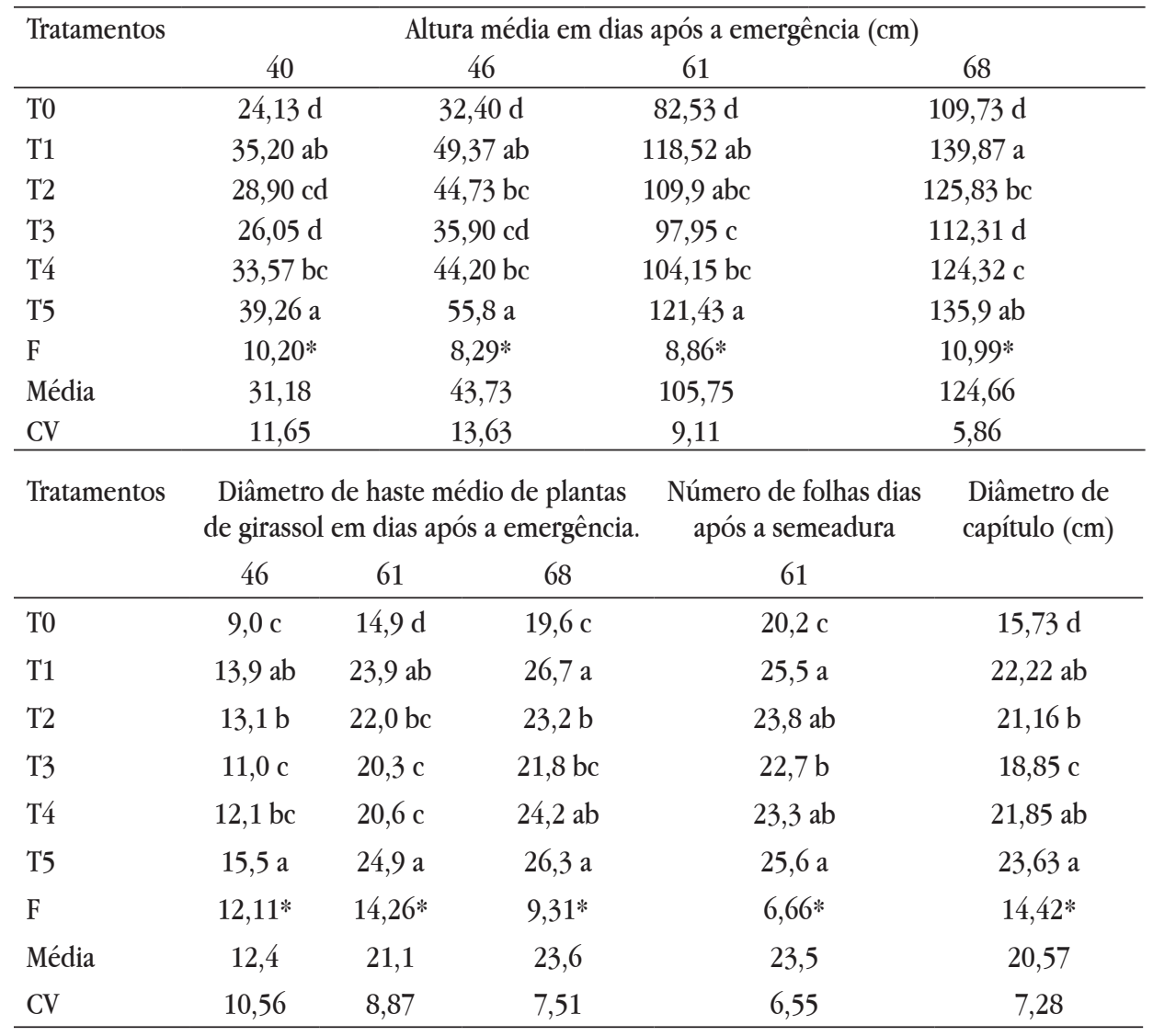

Verifica-se na Figura 4 que para todos os momentos com o aumento da dose de lodo compostado houve um aumento linear nas alturas das plantas.

Segundo Carvalho e Pissaia (2002), estudando dosagens de N (0, 25, 50, 75, 100 e 125) em kg ha-1 em girassol, não verificaram diferença em altura em nenhum dos momentos analisados. Entretanto este ensaio foi feito em plantio direto, em sucessão à soja, e a soja tem grande capacidade de fixar o $\mathrm{N}$ atmosférico através de bactérias em simbiose com a planta, então se acredita que o $\mathrm{N}$ fixado seja suficiente para a cultura do girassol.

Observa-se na Tabela 3 que o diâmetro médio de caule, onde não se aplicou $\mathrm{N}$ (T0), apresentou-se inferior em relação à aplicação de N, porém onde se aplicou 
$100 \%$ de $\mathrm{N}$ mineral (T1) e $200 \%$ de $\mathrm{N}$ de composto de lodo de esgoto (T5) foram superiores aos demais tratamentos na última medida e nas medidas anteriores este tratamento foi igual ao que recebeu $50 \% \mathrm{~N}$ mineral combinado com $50 \% \mathrm{~N}$ lodo compostado. Biscaro et al. (2008) obtiveram aumento do diâmetro do caule até a dose de 47,8 $\mathrm{kg} \mathrm{ha}^{-1}$ de $\mathrm{N}$, chegando a um crescimento médio do caule 18,4 mm. Verifica-se na Figura 4 um aumento linear do diâmetro de haste em função do aumento da dose de lodo compostado. Lobo e Grassi Filho (2009) obtiveram o maior diâmetro de haste com o aumento da dose de lodo de esgoto na cultura do girassol.

Observa-se na Tabela 3 que onde não foi aplicado N (T0) apresentou-se um menor número de folhas do que os demais tratamentos e dentre os tratamentos que foram aplicados $\mathrm{N}$ onde se aplicou 100\% $\mathrm{N}$ composto de lodo de esgoto foi inferior em relação onde se aplicou 100\% N mineral e 200\% N composto de lodo de esgoto. Biscaro et al. (2008) avaliaram que o número de folhas de girassol aos 45 dias de emergência houve um aumento linear em função do $\mathrm{N}$ aplicado até a dosagem máxima aplicada de $80 \mathrm{~kg} \mathrm{ha}^{-1}$ de $\mathrm{N}$. Verifica-se na Figura 4 que o aumento da dose de lodo proporcionou um aumento linear no número de folhas do girassol.

O diâmetro médio do capítulo tem implicações sobre o número do potencial de grãos, componente importante na produtividade de grão. Observa-se na Tabela 3 que onde não foi aplicado $\mathrm{N}$ apresentou-se inferior em relação onde foi aplicado N. Dentre os tratamentos que se aplicou N os tratamentos que foram suplementados com N mineral (T1 e T2) e 200\% N lodo compostado (T5) apresentaram superiores ao tratamento que foi aplicado somente o lodo compostado (T3) e o aumento da dose de lodo proporcionou um aumento do diâmetro do capítulo conforme mostra a Figura 4.

O N disponível nas fases iniciais pode afetar o desenvolvimento do capítulo, em razão de alterações celulares nos tecidos reprodutivos nos primeiros períodos de desenvolvimento, quando é determinado o número potencial de flores por capítulo, evidenciando aumento mesmo com doses pequenas ( $\left.25 \mathrm{~kg} \mathrm{ha}^{-1} \mathrm{de} \mathrm{N}\right)$. Porém, esse aumento não é contínuo com o aumento do N (SAMENI et al., 1976).

Lobo e Grassi Filho (2007) também obtiveram diferença no diâmetro de capítulo no tratamento que não foi aplicado $\mathrm{N}$ em relação ao que foi aplicado $\mathrm{N}$, comprovando que o $\mathrm{N}$ influencia no diâmetro de capítulo do girassol. 
A.

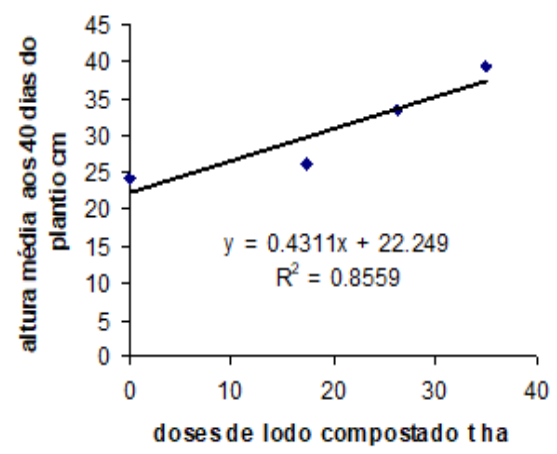

C.

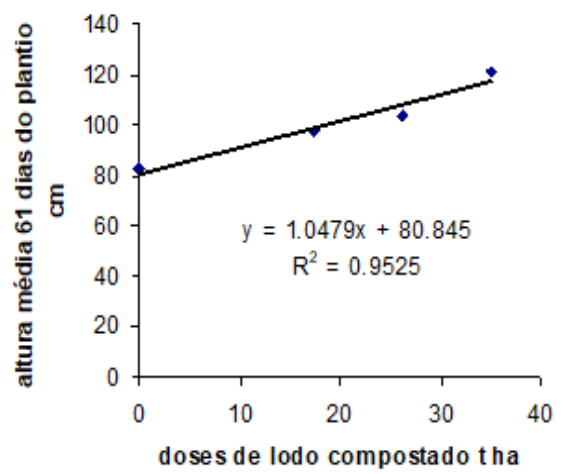

E.

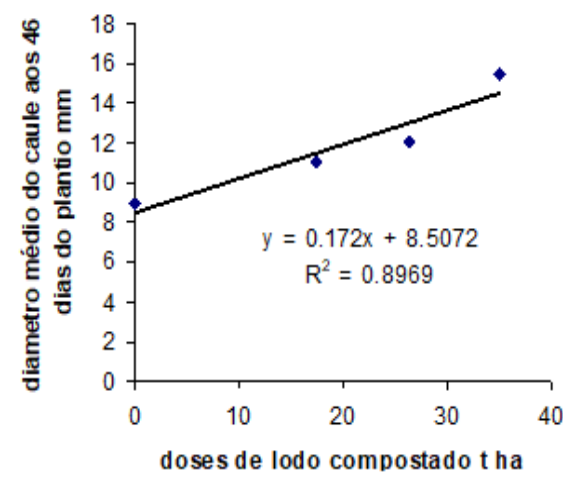

B.

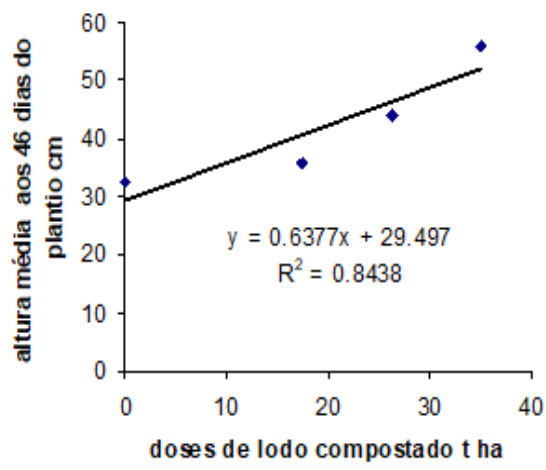

D.

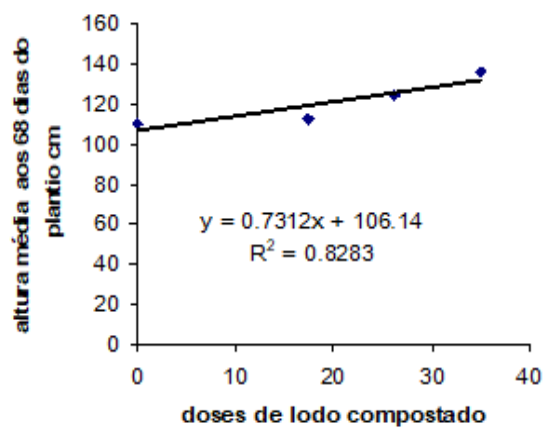

$\mathrm{F}$.

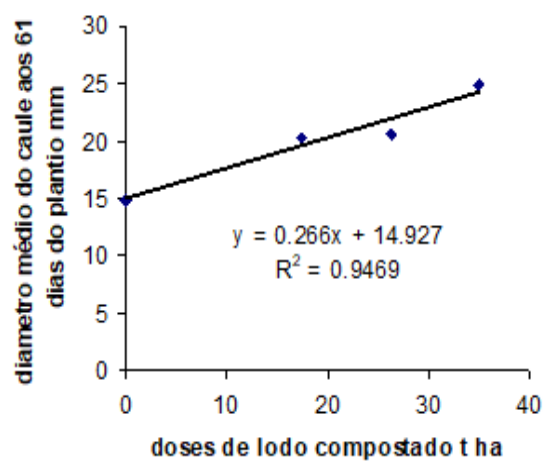


G.

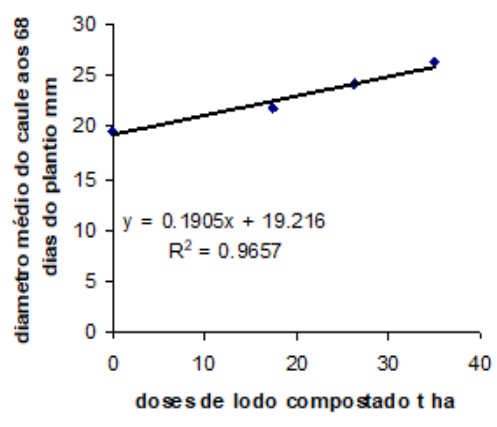

I.

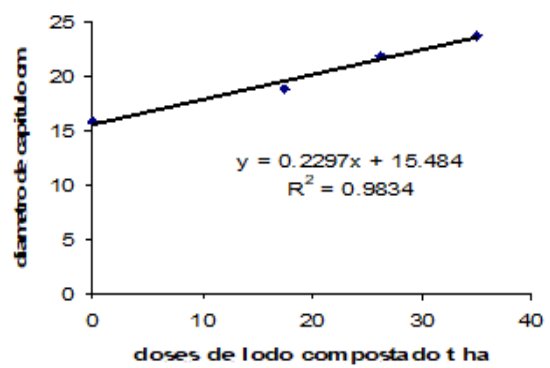

$\mathrm{H}$.

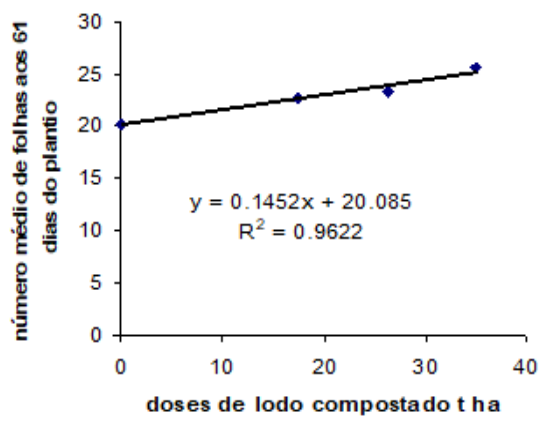

Figura 4. Altura de plantas no desenvolvimento do girassol em função da dose de lodo compostado em 40 (A), 46 (B), 61 (C) e 68 dias (D). Diâmetro médio de caule de plantas no desenvolvimento do girassol em função da dose de lodo compostado em 46 (E), 61(F) e 68(G) dias. Número médio de folhas aos $61(\mathrm{H})$ dias da semeadura em função da dose de lodo compostado. (I) Diâmetro médio de capitulo.

Fonte: Elaborada pelos autores

\section{CONSIDERAÇÕES FINAIS}

$\mathrm{O}$ aumento da dose de lodo de esgoto compostado proporcionou um incremento nos teores foliares de N, P, S, Fe, Mn e Zn e uma maior exportação de todos os nutrientes.

$\mathrm{O} \mathrm{N}$ proporcionou um decréscimo de K e um aumento do B, Fe e Mn na folha e um aumento na exportação de todos os nutrientes.

$\mathrm{O}$ aumento da dose de lodo de esgoto compostado e o $\mathrm{N}$ proporcionaram um aumento na altura de plantas, diâmetro de haste, número de folhas e diâmetro de capítulo. 


\section{REFERÊNCIAS}

ANJOS, J. L. DOS; SOBRAL, L. F.; CARVALHO, H. W. L. DE; OLIVEIRA, I. R. DE; CARVALHO, B. C. L. DE. Nitrogênio e fósforo na produção do girassol em solos dos tabuleiros costeiros. In: SIMPÓSIO NACIONAL SOBRE A CULTURA DO GIRASSOL, 6., Pelotas. Anais... Pelotas: Embrapa Soja, 2009. p. 149-153.

BETTIOL, W.; CAMARGO, O. A. Lodo de esgoto na agricultura: potencial de uso e problemas. TEC Hoje, v. 1, p. 1-4, 2007.

BISCARO, G. A.; MACHADO, J. R.; TOSTA, M. da S.; MENDONÇA, V.; SORATTO, R. P.; CARVALHO, L. A. de. Adubação nitrogenada em cobertura no girassol irrigado nas condições de Cassilândia - MS. Ciência Agrotecnológica, Lavras, v. 32, n. 5, p. 1366-1373, set./out., 2008.

BOEIRA, R. C. Uso do lodo de esgoto como fertilizante orgânico:

disponibilização de nitrogênio em solos tropical. Jaguariúna: EMBRAPA - Meio Ambiente, 2004. 5p. (Comunicado Técnico, n. 12).

CANTARELLA, H. Adubação e calagem do girassol. Sunflower response to lime and boron. Proceeding of the XI International Sunflower Association., 1985. p. 209215.

CARVALHO, D. B.; PISSAIA, A. Cobertura nitrogenada em girassol sob plantio direto na palha I rendimento de grão e seus componentes, índice de colheita e teor de óleo. Scientia Agrária, v. 3, n. 1-2, p. 41-45, 2002.

CASTRO, C. DE; OLIVEIRA, F. A. DE. Nutrição e Adubação do Girassol. In: CASTRO, C. de. Girassol no Brasil. Londrina: Embrapa SOJA, 2005. cap. 13, p. 317- 374. Companhia Nacional do Meio Ambiente. Resolução no 375/2006. 29 set. 2006. Disponível em: < http://www.mma.gov.br/post/conama/legiano/>. Acesso em: out. 2015.

CORREA, J. C.; BULL, L. T.; CRUSCIOL, C. A. C.; TECCHIO, M. A. Aplicação superficial de escoria, lama cal, lodos de esgoto e calcário na cultura da soja. Pesquisa Agropecuária Brasileira. v. 43, p. 1209-1219, 2008.

DECHEN, A. R.; NACHTIGAL, L. Micronutrientes. In: NUTRIÇÃO mineral de 
plantas. Viçosa: Sociedade Brasileira de Ciência do Solo, 2006. cap. 13, p. 327-354.

LOBO, T. F.; GRASSI FILHO, H.; BULL, L. T.; KUMMER, A. C. B. Efeito do lodo de esgoto e do nitrogênio nos fatores produtivos do girassol. Revista Brasileira de Engenharia Agrícola e Ambiental, v. 17, p. 504-509, 2013.

LOBO, T. F.; GRASSI FILHO, H. Sewage sludge on the development and nutricion of sunflower plants. Journal of Soil Science and Plant Nutrition, v. 9, p. 245255, 2009.

LOBO, T. F.; GRASSI FILHO, H. Níveis de lodo de esgoto na produtividade do girassol. Journal of Soil Science and Plant Nutrition, v. 7, p. 16-25, 2007.

MALAVOLTA, E.; VITTI, G. C.; OLIVEIRA, S. A. Avaliação do estado nutricional das plantas: princípios, métodos e técnicas de avaliação do estado nutricional. 2. ed. Piracicaba: Potafos, 1997. 319p.

RAIJ, B. V.; CANTARELLA, H.; QUAGAIO, J. A.; FURLANI, A. M. Recomendações de adubação e calagem para o Estado de São Paulo. 2. ed. Campinas: IAC, 1997. 198p. (Boletim Técnico, 100).

SAMENI, A. M.; MAFTOUN, M.; HOJJATTI, S. M.; SHEIBANY, B. Effect of fertilizer-N and herbicides on the growth and $\mathrm{N}$ content of sunflower. Agronomy Journal, Madison, v. 68, p. 285-288, 1976.

Recebido em: 27/09/2016 Aceito em: 24/05/2018 